\title{
QUELQUES CONSIDÉRATIONS LIMNOBIOLOGIQUES
}

\author{
Par M. J.-A. LESTAGE
}

Directeur de l'Aquarium de Bruxelles.

Parce que mes fonctions m'obligent à tripoter dans l'eau et, sans doute, jarce que l'eau est le domaine du Poisson, bien des pisciculteurs en herbe vier. nent me trouver et me posent cette question : "J'ai un étang. Je voudrais y mettre des Truites. Quel nombre de Poissons puis-je déverser?"

C'est effrayant ce qu'il pousse de saimoniculteurs 1 Je crois bien que neuf sur dix reculent devant les frais, mais if est curieux, pourtant, de voir cómbien de personnes, d'ailleurs sensées, se figurent qu'il suffit d'avoir de l'eau pour y metire des 'Truites et immédiatement gagner des sent et des mille!

- Que faites-vous, leur dis-je, quand vous voulez metire du bétail en pâture? Vous évaluez la grandeur de votre prairie, vous estimez ce qu'elle renferme d'herbe, vous supputez ce que chaque tête ent yourra manger, et vous êtes documenté sur le nombre d'animaux que vous pouvez parquer. C'est la méthode qu'il faut appliquer pour le Poisson. Vous avez de l'eau ? Que vaut cette eau? Documentez-vous sur la iempérature. Ic débit, le pH, pour savoir si le milieu conviant en lui-mème. Recherchez ensuite si le milieu est riche ou paúvre en nourriture, en quel genre de nourriture. Un ítang à Truites n'est pas un étang à Carpes. Ce qui convient pour l'un de res Poissons convient moins ou pell pour l'autre, surtout au premier âge. Que voulez-vous que vos alevins de Truites fassent devant des Trichoptères enclos dans leurs fourreaux de bois ow de pierres $P$ Que voulez-vous que vos Truites adultes fassent de l'unique plancton? Il faut a manger pour tout le monde, si vous ne nourrissez pas artificiellement, et il faut savoir quelle Truite accepte la nourriture artificielle.

Je sais bien que beaucoup de personnes n'ont cure de ce c fatras " scientifique, comme ils disent. Mais, pour quelques-uns dont l'empirisme n'a pas trompé les espoirs, il y en a cent dont les espérances ont été déçues. Et, alors, ce sont des plaintes multiples. On parle de la faillite des empoissonnements, de l'inutilité des teutatives de revalorisation. On ne songe pas que ces tentatives on à l'eau, ni au Poisson, mais au réempoissonneur. 


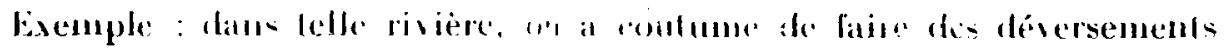
a tel endroil. Pour quelle faisen! Parlois un lignore: somvent a est a "ause de la facilite. Les bords smil accessibles! Pas de buissons encombrants ! Jolie plage ponr la manipulation des bidons, etc.! Tout semble farfait. C'est peut-être vrai; ce ne l'est peut-être pas du lout. Ici, romme ailleurs, le mieux peut ètre l'enmemi du bien.

Foin des plages faciles. Ne peusons pas à nuus, mais au Poisson. Trou\%-moi un emplacement plutòt tranquille, mais, surtout, riche en végólam immergés : Callitriches, Remuncules, Polamots, Myriophyles... Dans ve milieu calne, bos alevins éperdus ne seront pas saisis brusquement par un courant qui peut atre menrtrier. Dans es massifs phytiques. wos .ifevins trouveront abri et proics multiples.

On a peur, trop peur, de ces "berbes ", terrear du pècheur. Je les ai vu arracher, comme jia vil curer a's caux ol les trinsformer en baignoires.

Ici, peur et ignomance tolale. Ghaque lois que jen ai eu l'occasion, jai montré la splendide richesse jui peuple ces : herbes n, notamment ces mrnus Trichoptères et cos Ephémiroptères inmombrables, projes adaptées précisément à l'appétit des Truilelles déversécs. Mais ceux-là seuls sonl ronvaincus qui unt $v u$, et bien peu ont vu. Te sais des naturalistes, des professeurs, des pisciculteurs, je se parle pas des forestiers, connaisseurs " livresques " des organismes dulcicoles, qui les voyajent pour la première fois vivants. Récemment, jexphurais le Viroin, jolie rivière salmonicole ỵui nous vient de France. J'étais aver des pêcheurs de Truites, des as. Ils m'apportaient ailloux. plantes. sur lesquels se fouvaient les larves dont is voulaient savoir les woms of dudier l'imilation par les fabricants de. mouches $n$. lar hasard, je pris whe bratcheth. Jongue de trois doigts sur laquelle étaient accrochés par lours tils de soie des dizaines de fourreaux -ableux du joli Trichoptère qui a nom Oligoplectrum maculatum. L:1 rivière en contenait des milliards, et mes pêcheurs avaient pris ces fourreaux pour des brindilles!

In autre jour, explorant l' $\mathrm{i}$ isne. riche en Truites el surtout en Ombres, wyec le personnel forestier, j'espaçais mes hommes tous les 100 mètres. iver mission de récolter tout ce qu'ils trouveraient. Je constatai la prísence, partout, de millions de fourreaux de Glossosoma. Aucun de mes hommes ne les avait vus: ils les prenaient pour des graviers accolés aux ṇierres.

Donc, dạis les deux cas, c'était la méconnaissance d'un élément sitoinétrique de grande valeur ef d'une abondance prodigieuse. Pour les non initiés, c’étaient des eaux paures; pour le connaisseur, c'étaient des 'aux riches.

Dans un article récent ( $\mathrm{I}$ ), on signale la larve de Palingenia longirauda $\mathrm{OL}$. Or. non seulemen! l'animal ne ressemblc pas du tout à la

(1) Voir Bulletin, Mai ıgî. p. 33 r 
figure qui en est donnée, mais il ne doit plus exister en France, comme il a disparu en Belgique et en llollande. Ii cùt micux valu figurer un Ephéméroptère archicommun, de grande valeur, comme uti Cloeon ou un iraetis dont on capture les nilliers sur toutes le's plantes immergées.

Faute, donc, de bicn connaître tous les organismes constituant !a sitèse d'une eau, on pourra voir le gros, ce rlui houge, ce qui ne peui échapper à l'œil, mais on ne verru pas ce qui est fondamental souvent : le petit, l'immobile, l'étrangemcnt camoullé, comme les innombrables Hydroptilides, les Glossosoma, Agapclus, Oligoplectrum, Goera, Silo, etc., parmi les Trichoptères, et les larvules des Ephéméroptères.

On laissera même passer de grosses larves à cause de l'étrangeté de lcurs fourreaux, áinsi que je l'ai maintes fois constaté pour les Trichoplères suivants : - 1gry!onia pagetana, qui remplatce son fourreau spiralé par un fragment de Roseau; - Grammolaulius atorrarius, dont l'étui est formé de fragments végétaux assenıblés côte à côte ; - Glyphotaelius pel. lucidus, dont la maison a l'air d'ur paquet de petites feuilles; - et surtout Molanna et Molannodes, qui sont logés dens des amas sableux en forme de boucliers. Dix fois sur dix j'ai vu ces organismes, de belle taille pourtant, jetés hors du filet comme de simples "saletés".

Que valent, dans ce cas, les expertises biologiques?

Jutre chose ! Une eau est riche en organismes multiformes et en Mollusques. J'ai maintes fois vu des geus rejeter ces derniers ccmme des animaux sans valeur. Je connais des auteurs, au 'ourant pourtant de l'importance du problème sitométrique, dire atu sujet des Mollusques que c'est une nourriture secondaire, ou mème une nourriture de famine. Je ne lai jamais pensé. Au contraire, il m'a toujours parı que les Truites en étaient fort friandes, même quand elles avaient le choix. J'ai prié un de mes amis, Inspecteur des Eaux et Forêts et pècheur de Truites émérite, de bien vouloir examiner le tube digestif de toutes selles qu'il capturerait dans le Bocq, rivière fort riche en nourriture diverse. Les réponses étaient invariables : "Les Truites étaient bourrées de Mollusques ". Voilà donc un Gément de la capacité biogénique dont on aurait tort de sous-estimer l'importance.

Et il y a encore les Perlides, les Diplines, surtout lidmirable larve d'Atherix, et combien dautres encore.

Seulement, ce n'est pas tout de chercher; il laut savoir où chercher , it faut arriver à connaitre une eau au point de pouvoir dire ce que l'on y trouvera et où on le trouvera, et ce que l'on n'y peut pas trouver.

Evidemment c'est long̨, difficile, mais c'est nécessaire. Il n'est pas indispensable de connaitre le nom scientifique de tous ces animaux; mais il faut au moins connaître ces animaux, savoir ce qu'ils sont, ne pas !es tenir pour de simples détritus organiques ou autres, comme c'est souvent le cas.

Et puis, il convient de ne pas se laisser hypnotiser par le "plancton". 
Un croit vous fermer la bouche immédiatement avec ce mot. "Plancton ", voilà l'universelle panacée. Én euu calme, en élangs, dans les lacs, peutêtre, à la condution que soient ró:lisćes les conditions lavorables à la colonisation : apparition des Proto\%oaires ct des Bactéries, degré inférieur de la colonisation, qui préparent le terrain pour une foule d autres êtres qui feront leur nourrilure des pumiers habilants. Tout cela se passe fort bien dans les bassins tranquilles, où les sociélés lacustres peuvent se développer plus ou moins rapidement. C'est une erreur de supposer pareille chose dans les lrassins dont les eaux sont soumises à un continuel renouvellement, et, a fortiori, dans les caux quilifiées de salmonicoles. comme c'est le cas pour les rivières et ruisscaux rapides. C'est peut-être !'origine de la fameuse dispute historique entre les partisans et ies détractcurs du plancton, parce que les uns l'étudiaiont dans des milieux léniliques, les autres dans des milıeux lotiques.

"Il est difficile d'admeltre, - écrivaient J. Porrien et C. Bruyant (I), qu'une Truite de ró kilos, conme on en pèche au Pavin, s'alimente exclusivement avec les ètres meroscopiques qui conslituent le plancton :. Je le crois sans peine. Il était facile de s'en convitincre par le seul examen Ju contenu du tube digrestif. La réponse cût été imnédiate et péremptoire.

Il est mìme au moins trop tòt pour parler de polamoplancton. Existe$t$-il en réalité un plancton fluvial rrai ?

Luis Pardo écrivait en 1924, à propos de la prélérence montrée pour !'étude du limnoplancton plutồ que pour cel!e lu potamoplancton, que " celte prélérence est logique, si l'on considère que la vie, dans les lacs, est bien plus riche que dans les fleuves "(2). Il est vrai que Meissver (3) connaissait 24 Entomostracés dans la Volga, Koford (4) 500 dans l'Illinois, ¿ Volk (5) x .000 environ dans l'Elbe. Mais où niche ce plancton potamobionte? Dans des biotopes à facics essentiellement limnétique, riches "n matières organiques dissoutes, donc dans les zones calmes des bords, ià où la végétation détermine la richesse plancionique. Les recherches do Pardo dans la Jucar lu firent trouver cinq organismes du phytoplancton et trois du zooplancton, les premiers appartenant aux groupes des Diatomées et des Conjuguées, les seconds aux Cladocères et Cepépodes.

Souvent on suppose que "lcs luisseaux ì cau claire et un peu torrentielle donneront un apport spécial $"$. C'est possible; mais, espérer que ce 'jui existe, et ce qui pourra peut-itre cxistcr, suffira à faire vivre (je ne dis pas engraisser) les Poiston", c'est um: autre affairc.

Il ne suffit pas de dire qu une chose existe, il le faut prouver. Pour !e prouver, il faut connaître ce dont on parle. Pour en parler, il le faut étu-

(ז) Annales de Biologie lacustre, I, x go6, p.:6.

(2) Pardo, L. : - Notes sur le polamoplanclon de Valencia (Espagne). - Annales de Biologie lacustre, XIII, 192\%, p. $9^{3}$.

(3) Mrissver, A. :-Zoologischer Anzeiger, XXVI, 1902.

(4) Koford, C.-A. : - Bullelin of lllinois Labor. Natur. History, VI-VIII, 19o3-igo8.

(5) Vouk, R. : - Mitlcilungen aus dem natur. Museum. Hamburg, XIX-XXXIII, $1903-1906$. 
dier. Or, je crois que les doigts d'une seule main suffisent pour compter reux qui, au moins en Belgirque, ont étudié les organismes dulcicoles composant la sitèse fondamentale des caux.

C'est peu, et, pourtant, c'est l'cuure jatienic de ces hommes qui seule nous donnera la solution du problème. Il suffit de voir les travaux d'un LÉger, d'un Rocle, d'un Ilesse, d'un p'aris, d'un llubaclt, pour se rendre comple de la nécessité des recherches hydrobiologiques. Puissent-ils nous donner une brillante pléiade d'élèves qui sauront revaloriser noz eaux par la seule méthode qui corvient!

\title{
NOUVEAUX DISPOSITIFS POUR INTERDIRE AUX POISSONS L'ACCĖS DES DÉRIVATIONS HYDRAULIQULS
}

\author{
PAH M. C. GAILLUIS \\ Cincservaleur des Eatux ol Fords on retraite. \\ et M. DE DROLIN DE BOUVILLA: \\ Inspecteur principal des Futux el Fords all relrails.
}

(Fin) ( 1 )

Ces indications générales données sur la siluaticn, telle qu'elle existait au commencement de l'annéc dernière, nous emplunterons au second

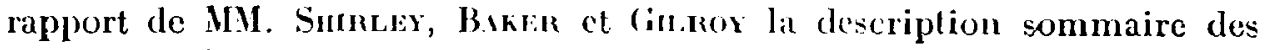
điverses grilles électriques alor's en strvice, a lonest des Etats-Lnis, sur ijuatre emplacements différents.

Sunnyside Caral. - Le canal de Sumnside, maitresse artère du réseau de la Yakima (Yalima Project, l. S. Reclamalion Service), emprunte les caux de la rivière de ce nom, aflílucut de droit: de la Columbia, près de la ville appelée également Yahima! Washingloul.

La saison d'irrigation, (n rgeg, a commence le. 15 Mars pour linir ie: $\because 1$ Octobre. Durant la majeure partie de cette campagne, le débit a oscillé entre 33.600 et 42.000 litres-seconde.

A l'emplacement du barrage électrique, cn anon: des vannes d'admission, le canal a une largeur de $15 \mathrm{~m}$. $25 \mathrm{ct}$ une profondeur de $2 \mathrm{~m}$. 13. Ce barrage consiste en un rideau d'électrodes formées Je cheînes galvanisées longues de $9 \mathrm{r} \mathrm{cm}$. I/2, espacées de $3-\mathrm{cm}, 1 / 2$, suspendues à un câble de support tendu d'une rive à l'autre un peu au-dessus de l'eau. Sur le fond

(1) Voir Bulletin, Septembre rg3r, р $7 ;$; Novembre igâr, p. r33. 